\title{
Association between the Epidermal Growth Factor 61*A/G Polymorphism and Hepatocellular Carcinoma Risk: a Meta- Analysis
}

\author{
Shuang Sun ${ }^{1 \&}$, Guo-Jiang Jin ${ }^{1 \&}$, Yan Zhao², Hui Kang ${ }^{1 *}$
}

\begin{abstract}
The epidermal growth factor (EGF) may play a pathological role in hepatocellular carcinoma (HCC). However, the conclusions of published reports on the relationship between the EGF $61 * \mathrm{~A} / \mathrm{G}$ polymorphism and HCC risk remain controversial. To derive a more precise estimation we performed a meta-analysis based on 14 studies that together included 2,506 cases and 4,386 controls. PubMed, EMBASE, Web of Knowledge and the Chinese National Knowledge Infrastructure (CNKI) databases were used to retrieve articles up to August 1, 2014. The crude odds ratios (ORs) with $95 \%$ confidence intervals $(95 \% \mathrm{CIs})$ were calculated to evaluate the association. Meta-analysis results showed a significant association between the EGF $61 * \mathrm{~A} / \mathrm{G}$ polymorphism and HCC risk in all four genetic models (allele model: $O R=1.25,95 \% \mathrm{CI}=1.12-1.40$; dominant model: $\mathrm{OR}=1.32$, 95\% CI=1.14-1.54; recessive model: $\mathrm{OR}=1.33,95 \% \mathrm{CI}=1.12-1.58$; ho-mozygous model: $\mathrm{OR}=1.59,95 \% \mathrm{CI}=1.33$ 1.90). Moreover, significant associations were observed when stratified by ethnicity, source of controls, etiology and genotype methods. Thus, this meta-analysis suggests that the G-allele of the EGF $61^{*} \mathrm{~A} / \mathrm{G}$ polymorphism is associated with an increased risk of $\mathrm{HCC}$, especially in Asians and Caucasians, without influence from the source of controls or etiological diversity. Further studies with larger population sizes are needed to confirm these results.
\end{abstract}

Keywords: Hepatocellular carcinoma - polymorphism - meta-analysis - EGF $61 * A / G$

Asian Pac J Cancer Prev, 16 (7), 3009-3014

\section{Introduction}

Hepatocellular carcinoma (HCC) is the fifth most common malignancy and the third most lethal type of cancer worldwide (El-Serag, 2011). Cirrhosis related to al-cohol or chronic hepatitis B virus (HBV) and hepatitis $\mathrm{C}$ virus (HCV) infections are major risk factors in $\mathrm{HCC}$ carcinogenesis. However, only a fraction of infected patients develop HCC; therefore, genetic alterations are also thought to play critical roles in HCC pathogenesis (El-Serag et al., 2007; Chuang et al., 2009; Yue et al., 2013). Recent studies have demonstrated that modulation of molecular signaling pathways occurs in malignant transformation of hepatocytes and HCC progression (Llovet et al., 2008; Zender et al., 2010).

The epidermal growth factor (EGF) gene is a member of the EGF superfamily. It is located on chromosome 4q2527. As an endocrine growth factor, EGF performs a key role in promoting cell survival, activating DNA synthesis and it is also an im-portant factor for proliferation and differentiation of epithelial cells (Lanuti et al., 2008). EGF is commonly overexpressed in human cancers, such as glioma, pancreatic, breast and gastrointestinal cancer, suggesting an important role in malignant cell transformation, tumor occurrence, and development by promoting cell division (Sto-scheck et al., 1986; Nicholas et al., 2006). EGF also plays a critical role in the occurrence of liver cancer via binding to the EGF receptor (EGFR) and activating intracel-lular signal transduction pathways (Jorissen et al., 2003). EGF has a functional sin-gle-nucleotide polymorphism at position 61 of the 5:-untranslated region involving the substitution of adenine (A) for guanine $(\mathrm{G})(61 * \mathrm{~A} / \mathrm{G}$, rs4444903). Homozygous $61 \mathrm{~A}$ allele carriers have lower levels of EGF expression than $61 \mathrm{G}$ homozygous or A/G heterozygous individuals (Shahbazi et al., 2002). Thus, this genetic polymorphism may contribute to interindividual differences of EGF expression and subsequently tumor predisposition and aggressiveness.

A wide variety of studies have reported association between the EGF $61 * \mathrm{~A} / \mathrm{G}$ polymorphism and susceptibility to HCC; however, these studies have produced inconsistent results (Tanabe et al., 2008; Qi et al., 2009; Wang et al., 2009; Li et al., 2010; Abu Dayyeh et al., 2011; Chen et al., 2011; Abbas et al., 2012; Shi et al., 2012; Wu et al., 2013; Suenaga et al., 2013; Yuan et al., 2013). 
Shuang Sun et al

Therefore, we performed a meta-analysis of the 14 most recent and relevant case-control studies involving 2, 506 cases and 4, 386 controls to further evaluate the precise association of the EGF $61 * \mathrm{~A} / \mathrm{G}$ polymorphism with HCC risk, as well as to provide a clinical reference and a basis for HCC treatment.

\section{Materials and Methods}

\section{Publication search}

We performed a systematic search for eligible casecontrol studies in PubMed, EMBASE, Web of Knowledge and the Chinese National Knowledge Infrastructure (CNKI) databases up to August 1, 2014. A combination of the following search phrases were used: "EGF" (or "epidermal growth factor"), "polymorphism" (or "variant"), and "HCC" (or "hepatocellular carcinoma" or "liver cancer"). There was no limitation in the publication search, and reference lists were examined manually to further identify potentially relevant studies.

\section{Selection Criteria}

Studies included in the meta-analysis were required to meet the following criteria: (1) full-text articles; (2) casecontrol studies that evaluated the association between the EGF $61 * \mathrm{~A} / \mathrm{G}$ polymorphism and $\mathrm{HCC}$ risk; (3) provision of sufficient data about EGF $61 * \mathrm{~A} / \mathrm{G}$ genotypes and genotype distributions to estimate the odds ratios (ORs) with $95 \%$ confidence intervals (95\%CIs). If there were overlapping samples in dif-ferent publications, we chose the most recent study with the largest sample size or ex-cluded overlapping samples. Studies were excluded if one of the following existed: (1) irrelevant papers; (2) not case-control studies; (3) based on incomplete data; (4) letters, reviews, meta-analyses.

\section{Data extraction}

Two investigators independently extracted data according to the inclusion crite-ria listed above and reached a consensus on all of the items. For each study, the fol-lowing characteristics were collected: first author's surname, publication year, coun-try of origin, ethnicity, source of controls, sample sizes of cases and controls, number of genotypes, P-value for Hardy-Weinberg equilibrium (HWE), genotyping methods.

\section{Statistical analysis}

The strength of the association between the EGF $61 * \mathrm{~A} / \mathrm{G}$ polymorphism and HCC susceptibility was measured by ORs with $95 \%$ CIs under four genetic models, including the allele model ( $\mathrm{G}$ vs $\mathrm{A})$, dominant model $(\mathrm{GG}+\mathrm{AG} v s \mathrm{AA})$, recessive model (GG vs $\mathrm{AG}+\mathrm{AA})$ and homozygous model (GG vs AA). We used the $\chi^{2}$ test to assess the HWE of the genotype frequencies of controls and the significance was set as $\mathrm{P}<0.05$. The statistical significance of pooled ORs was determined with the Z-test and $\mathrm{P}<0.05$ was considered as statistically significant. Cochran's Q test and the $\mathrm{I}^{2}$ sta-tistical test were used to estimate potential heterogeneity across the studies (Higgins et al., 2002; Zintzaras et al., 2005). A fixed effects model was used when $\mathrm{P}>0.05$ in the Q test and
$\mathrm{I}^{2}<50 \%$ were determined simultaneously, while a random effects model was selected when $\mathrm{P}<0.05$ in the $\mathrm{Q}$ test and $\mathrm{I}^{2}>50 \%$ (Mantel et al., 1959; DerSimoni-an et al., 1986). The pooled ORs were first calculated according to both healthy group controls and controls with cancer-free liver diseases. To investigate the possibility of heterogeneity, we also performed subgroup analysis by ethnicity and genotype meth-od. Sensitivity was performed by omitting individual studies and re-calculating the ORs and the $95 \%$ CIs in order to assess the stability of results. Begg's funnel plots and Egger's linear regression test (significance level was set at 0.05 ) were performed to investigate potential publication bias. Analyses were calculated using Stata soft-ware version 12.0 (Stata Corp., College Station, TX, USA) and all $\mathrm{P}$ values were two-sided

\section{Results}

\section{Study characteristics}

Based on our search criteria, 12 publications relevant to the role of the EGF $61 * \mathrm{~A} / \mathrm{G}$ polymorphism in $\mathrm{HCC}$ susceptibility were identified. One of these articles was excluded, as two publications by Qi et al. (2008; 2009) were based on duplicate data, so they were considered as one study. Three publications (Tanabe et al., 2008; Wang et al., 2009; Yuan et al., 2013) each involved two independent case-control studies and were considered separately, giving six studies altogether. As a result, a total of 14 relevant studies comprising 2, 506 cases with HCC and 4, 386 controls were included in the meta-analysis (Figure 1). The main characteristics of the selected studies and the genotype distribution of the EGF $61 * \mathrm{~A} / \mathrm{G}$ polymorphism are summarized in Table 1. Among them, nine studies involved Asian subjects (Qi et al., 2009; Wang et al., 2009; Li et al., 2010; Chen et al., 2011; Shi et al., 2012; Wu et al., 2013; Suenaga et al., 2013; Yuan et al., 2013), two involved Caucasians (Tanabe et al., 2008; Abbas et al., 2012) and three involved mixed populations (White, Black, His-panic, Asian and other) (Tanabe et al., 2008; Abu Dayyeh et al., 2011; Yuan et al., 2013). Three studies involved Asian populations with unique HBV infection etiology (Qi et al., 2009; Li et al., 2010; Chen et al., 2011),

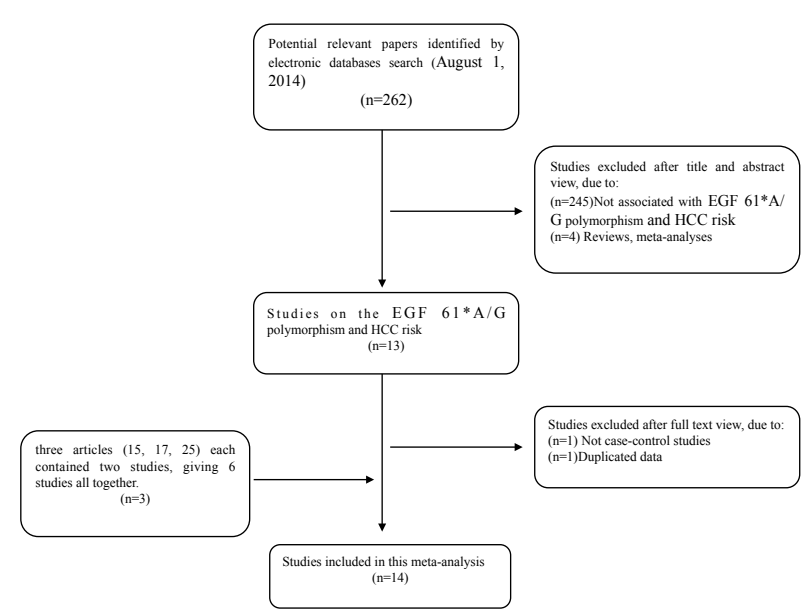

Figure 1. Flow Chart of Publication Selection Procedure 
three concerned Asian subjects with predominantly HCV infection (Abu Dayyeh et al., 2011; Abbas et al., 2012; Suenaga et al., 2013) and one study investigated solely alcohol-related HCC (Tanabe et al., 2008). The controls were mainly healthy populations, except in studies by Tanabe et al., Abu Dayeh et al. and Suenaga et al.. Moreover, four studies contained both healthy and HBV/ HCV infected controls (Qi et al., 2009; Li et al., 2010; Chen et al., 2011; Abbas et al., 2012). Several genotyping methods were used, including pol-ymerase chain reaction - restriction fragment length polymorphism (PCR-RFLP), TaqMan assay, and Allele-specific PCR. The distributions of the EGF $61 * \mathrm{~A} / \mathrm{G}$ geno-type among the control subjects were tested and all were in HWE.

\section{Meta-analysis results and heterogeneity analysis}

Evaluation of the association between the EGF $61 * \mathrm{~A} / \mathrm{G}$ polymorphism and $\mathrm{HCC}$ risk is presented in Table 2. Overall, significant main effects on HCC risk were ob-served in all four genetic models (allele model: $\mathrm{OR}=1.25,95 \% \mathrm{CI}=1.12-1.40$; domi-nant model: $\mathrm{OR}=1.32,95 \% \mathrm{CI}=1.14-1.54$; recessive model: $\mathrm{OR}=1.33$, 95\% CI=1.12-1.58; homozygous model: $\mathrm{OR}=1.59$, 95\% CI=1.33-1.90) (Figure 2). In sub-group analysis based on different ethnicity, significant risks were also found among Asians (allele model: $\mathrm{OR}=1.17,95 \% \mathrm{CI}=1.07-1.28$; dominant model: $\mathrm{OR}=1.25,95 \% \mathrm{CI}=1.04-1.49$; recessive model: $\mathrm{OR}=1.20,95 \% \mathrm{CI}=1.07-1.36$; homozygous model: $\mathrm{OR}=1.47,95 \% \mathrm{CI}=1.20-1.81$ ), Caucasians (allele model: $\mathrm{OR}=1.93,95 \% \mathrm{CI}=1.25-2.98$; recessive model: $\mathrm{OR}=3.07,95 \% \mathrm{CI}=1.48-6.35$; homozygous model: $\mathrm{OR}=3.52,95 \% \mathrm{CI}=1.50-8.62)$, and in Mixed populations under the dominant model $(\mathrm{OR}=1.52,95 \% \mathrm{CI}=1.08-2.16)$. Likewise, significant risk was observed in all genetic models whether on the basis of healthy controls (allele model: $\mathrm{OR}=1.15,95 \% \mathrm{CI}=1.05-1.26$; dominant model: $\mathrm{OR}=1.21,95 \% \mathrm{CI}=1.02-1.44$; recessive model: $\mathrm{OR}=1.19$, $95 \% \mathrm{CI}=1.05-1.35$; homozygous model: $\mathrm{OR}=1.38$, $95 \% \mathrm{CI}=1.12-1.70)$ or on the basis of controls with cancerfree liver diseases (allele model: $\mathrm{OR}=1.36,95 \% \mathrm{CI}=1.19$ 1.55; dominant model: $\mathrm{OR}=1.79,95 \% \mathrm{CI}=1.35-2.37$; recessive model: $\mathrm{OR}=1.55,95 \% \mathrm{CI}=1.15-2.10$; homozygous model: $\mathrm{OR}=2.26,95 \% \mathrm{CI}=1.66-3.07)$. In further stratified analysis with respect to etiology, a significant association in patients with $\mathrm{HBV}$ infection was observed in all genetic models. Similarly, significant relationships were observed in patients with $\mathrm{HCV}$ infection and alcoholic cirrhosis, ex-cept in the recessive and dominant models, respectively. In addition, a significant ef-fect of genotype method was observed for RFLP in all genetic models; however, no significant elevated risks were found for TaqMan assay and Allele-specific PCR un-der the dominant model.

We stratified the studies according to ethnicity and genotype method, to find the sources of heterogeneity among findings. The random effects model was used since the heterogeneity was obvious $(\mathrm{P}<0.05)$. In the overall comparison and subgroup analysis, we observed significant heterogeneity under the allele and recessive models, which might be due to the Mixed subjects and the RFLP genotyping method $(\mathrm{P}<0.05)$.

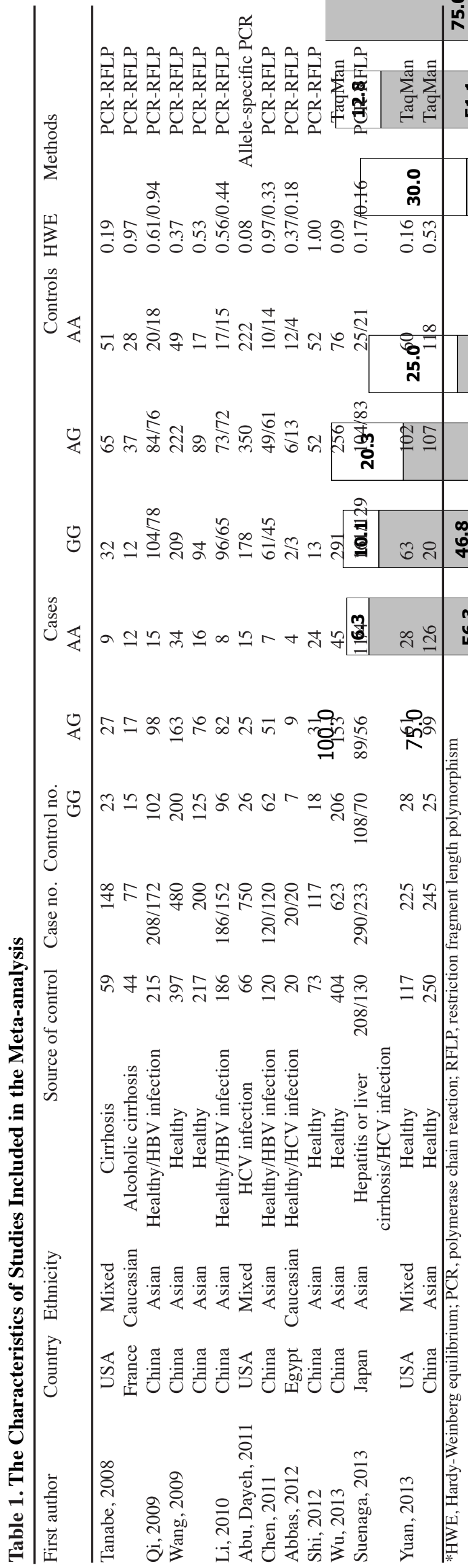

Asian Pacific Journal of Cancer Prevention, Vol 16, 2015 

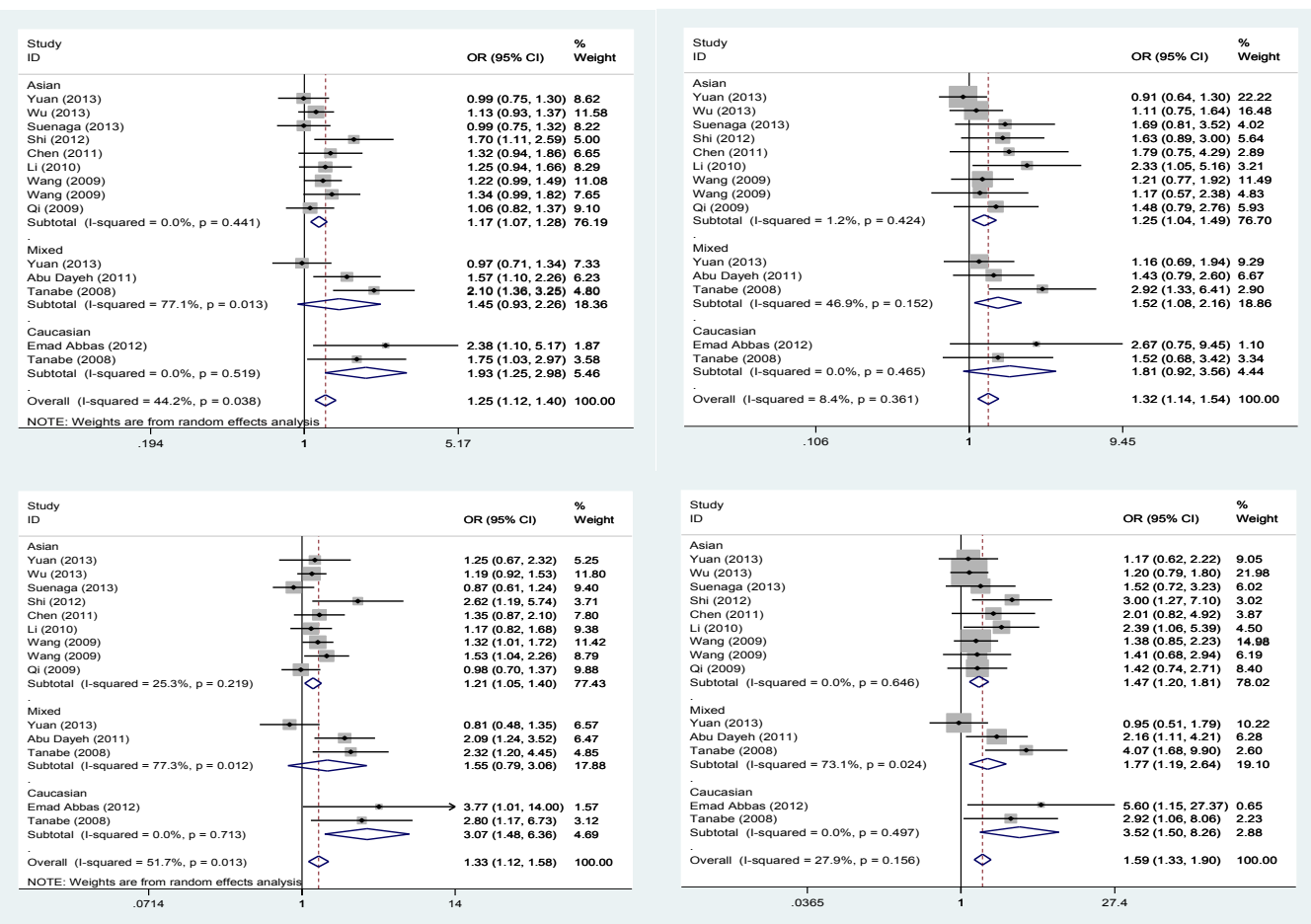

Figure 2. Forest Plot for the Relationships of EGF 61*A/G Genetic Polymorphisms and the Risk of HCC. A) Allele Model; B) Dominant model; C) recessive model; D) homozygous model)

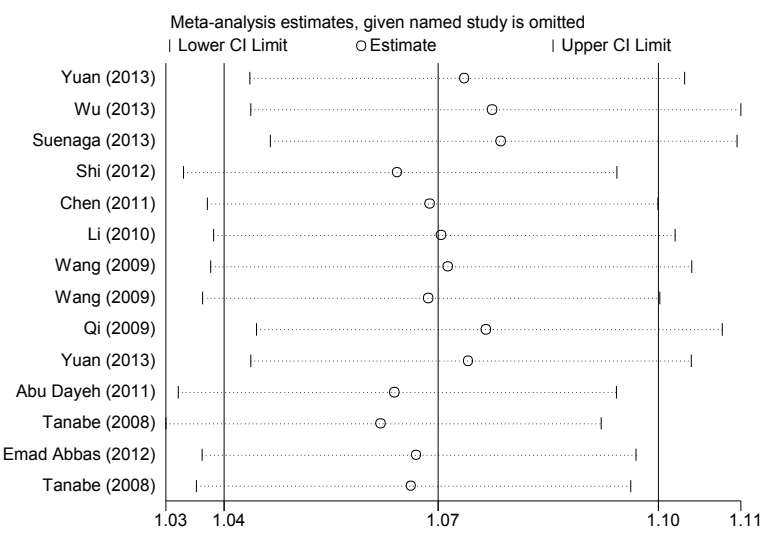

Figure 3. Sensitivity Analysis of the Association between EGF 61*A/G Polymorphism and HCC Under the Allele Model. The figure shows the influence of individual studies on the pooled odds ratio

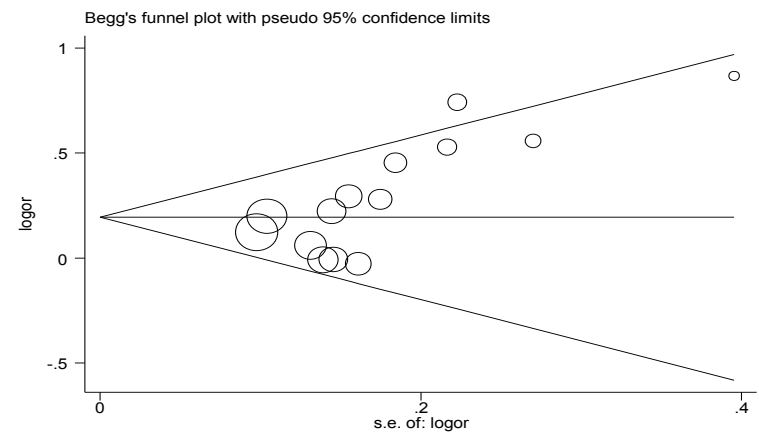

Figure 4. Beger's Funnel Plot of Publication Biases on the Relationships of EGF 61*A/G Genetic Polymorphism and the Risk of HCC Under the Allele

Model. Each point represents a separate study for the indicated association. $\log (\mathrm{OR})$, natural logarithm of odds ratio; horizontal line, mean effect size
Sensitivity analysis

Sensitivity analysis was performed to assess the influence of each individual study on the pooled ORs by omitting one study at a time. This analysis suggested that the significance of the pooled ORs under the allele model (G vs A) of EGF 61*A/G was not influenced excessively by omitting any single study (Figure 3 ), indicating that our results are statistically reliable.

\section{Publication bias}

Begg's funnel plot and Egger's test were conducted to access publication bias in this meta-analysis. The funnel plots of Begg's test showed some asymmetry (Figure 4) that was subsequently corroborated by Egger's test. There was evidence of publica-tion bias among all genetic models (allele model: $\mathrm{P}=0.006$; dominant model: $\mathrm{P}=0.000$; recessive model: $\mathrm{P}=0.023$; heterozygous model: $\mathrm{P}=0.001$ ).

\section{Discussion}

EGF is a potent mitogen for hepatocytes (Blanc et al., 1992) and contributes to liver tissue regeneration through binding to EGFR (Natarajan et al., 2007). EGF/EGFR signaling is dysregulated in early hepatocarcinogenesis and this supports autocrine growth stimulation of hepatoma cells (Yamaguchi et al., 1995; Chung et al., 2002). Therefore, overexpression of EGF might be a critical step toward development of HCC.

The EGF $61 * \mathrm{~A} / \mathrm{G}$ functional polymorphism in the gene promoter region was observed to modulate EGF levels and could thus increase the risk of HCC. Some studies have indicated that the EGF $61 * \mathrm{G} / \mathrm{G}$ genotype is associated with increased HCC susceptibility (Tanabe et al., 2008; Abu Dayyeh et al., 2011; Abbas et al., 2012; Shi et al., 2012), whereas other studies have not (Qi et al., 2009; Wang et al., 2009; Li et al., 2010; Chen et al., 2011; Wu 
et al., 2013; Suenaga et al., 2013; Yuan et al., 2013). The meta-analysis performed by Yang et al. determined that the EGF $61 \mathrm{G}$ al-lele is a risk factor for developing HCC without the influence of ethnic diversity, while Zhong et al. also showed that the EGF $61 * \mathrm{G}$ polymorphism is a risk factor for HCC, but especially in the Chinese population.

In our meta-analysis, on the basis of collecting more studies than previous analyses, we show that the $\mathrm{G}$ allele has an in-creased HCC risk compared with the A allele in the overall comparison, and that the association was more pronounced for all genetic models in Asians and Caucasians, but only in the dominant genetic model in the Mixed population. As the number of articles involving Caucasians and Mixed populations was limited in our meta-analysis, there might be selection bias in these two populations. Accordingly, the significance of the results should be interpreted with some caution. But for Asians, previous stud-ies by Qi et al. and Li et al. concluded that the East Asian population has a low AA genotype frequency and a high GG genotype frequency, which, together with our findings, may explain the higher HCC prevalence among the Asian population.

In addition, we divided control populations into two groups; healthy controls and controls with cancer-free liver diseases. We further confirmed the conclusion that the EGF $61 * \mathrm{~A} / \mathrm{G}$ genotypes are associated with increased HCC risk without any discrep-ancy from the source of controls. In contrast, Zhong et al. found that the EGF $61 * \mathrm{G}$ allele was statistically associated with increased risk of HCC among hospital-based controls, but not populationbased controls, and concluded that the polymorphism is a genetic susceptibility factor for HCC only in the background of chronic HBV infec-tion and/or cirrhosis. As hepatocarcinogenesis is a long-term multistage process with the involvement of multiple risk factors, functional studies that consider etiology, host genetic factors and environmental factors are required to fully explain its pathogenesis.

In the subgroup analysis by etiology, we demonstrated a significant risk between HBV-related HCC and the EGF $61 * \mathrm{~A} / \mathrm{G}$ polymorphism. Significant association was also found in HCV-related HCC and alcoholic cirrhosis-related HCC. These results indicate that the ability of the EGF $61 * \mathrm{~A} / \mathrm{G}$ polymorphism to contribute to HCC does not depend on different etiological factors. Only one study of alcohol cirrhosis-related HCC was included in the stratified analysis; therefore, the possibility of finding a re-liable association is limited. The statistical significance of the EGF $61 * \mathrm{~A} / \mathrm{G}$ variant with HCC risk suggests that this variant may be a potential biomarker for early diagnosis, prediction of patient outcome, or for the direction of optimal therapy for individual patients.

In interpreting the current results of our meta-analysis, some limitations should be considered. First, the pooled results are based on unadjusted OR estimates because not all eligible studies presented adjusted ORs. A more precise evaluation should be adjusted by potential confounders, such as age, sex, family history, environmental factors, and cancer stage. Second, potential interactions among gene-gene, gene-environment and even different genetic variations in EGF were not analyzed owing to the lack of relevant data. Finally, publication bias may exist as no attempts were made to identify unpublished articles. Despite the limitations of our analysis, our meta-analysis still has two advantages. First, we added seven recent studies that have not been included in previous metaanalyses; a substantial number of cases and con-trols were pooled from different studies giving significantly increased statistical pow-er. Second, the meta-analysis was conducted using rigorous methods of study selec-tion, data extraction, and data analysis.

In conclusion, our meta-analysis suggests that the G-allele of the EGF $61 * \mathrm{~A} / \mathrm{G}$ polymorphism is associated with an increased risk of HCC, especially in Asians and Caucasians, and that the associations were not affected by the source of controls or etiological diversity. Largescale studies with more detailed individual data of genegene and gene-environment investigations are needed to validate our results.

\section{References}

Abbas E, Shaker O, Abd El Aziz G, et al (2012). Epidermal growth factor gene pol-ymorphism $61 \mathrm{~A} / \mathrm{G}$ in patients with chronic liver disease for early detection of hepa-tocellular carcinoma: a pilot study. Eur J Gastroenterol Hepatol, 24, 458-63.

Abu Dayyeh BK, Yang M, Fuchs BC, et al (2011). a functional polymorphism in the epidermal growth factor gene is associated with risk for hepatocellular carcinoma. Gastroenterology, 141, 141-9.

Blanc P, Etienne H, Daujat M, et al (1992). Mitotic responsiveness of cultured adult human hepatocytes to epidermal growth factor, transforming growth factor alpha, and human serum. Gastroenterology, 102, 1340-50.

Chen K, Wei Y, Yang H, et al (2011). Epidermal growth factor $+61 \mathrm{G} / \mathrm{A}$ polymor-phism and the risk of hepatocellular carcinoma in a chinese population. Genet Test Mol Biomarkers, 15, 251-5.

Chuang SC, La Vecchia C, Boffetta P (2009). Liver cancer: descriptive epidemiology and risk factors other than HBV and HCV infection. Cancer Lett, 286, 9-14.

Chung YH, Kim JA, Song BC, et al (2002). Expression oftransforming growth fac-tor-alpha mRNA in livers of patients with chronic viral hepatitis and hepatocellular carcinoma. Cancer, 89, 977-82.

DerSimonian R, Laird NM (1986). Meta-analysis in clinical trials. Control Clin Trials, 7, 177-88.

El-Serag HB, Rudolph KL (2007). Hepatocellular carcinoma: epidemiology and mo-lecular carcinogenesis. Gastroenterology, 132, 2557-76.

El-Serag HB (2011). Hepatocellular carcinoma. New Engl J Med, 365, 1118-27.

Higgins JP, Thompson SG (2002). Quantifying heterogeneity in a metaanalysis. Statistics Medicine, 21, 1539-58.

Jorissen RN, Walker F, Pouliot N, et al (2003). Epidermal growth factor receptor: mechanisms of activation and signaling. Exp Cell Res, 284, 31-53.

Lanuti, M (2008). A functional epidermal growth factor (EGF) polymorphism, EGF serum levels, and esophageal adenocarcinoma risk and outcome. Clin Cancer Res, 14, 3216-22.

Li Y, Xie Q, Lu F, et al (2010). Association between epidermal growth factor $61 \mathrm{~A} / \mathrm{G}$ polymorphism and hepatocellular carcinoma susceptibility in Chinese patients. Liver Int, 30, $112-8$. 


\section{Shuang Sun et al}

Llovet JM, Bruix J (2008). Molecular targeted therapies in hepatocellular carcinoma. Hepatology, 48, 1312-27.

Mantel N, Haenszel W (1959). Statistical aspects of the analysis of data from retro-spective studies of disease. J Natl Cancer Inst, 22, 719 -48.

Natarajan A, Wagner B, Sibilia M (2007). The EGF receptor is required for efficient liver regeneration. Proc Natl Acad Sci USA, 104, 17081-6.

Nicholas MK, Lukas RV, Jafri NF, et al (2006). Epidermal growth factor receptor - mediated signal transduction in the development and therapy of gliomas. Clin Cancer Res, 12, 7261-70.

Qi P, Chen YM, Gao CF, et al (2008). Relationship between polymorphism of epi-dermal growth factor 59UTR Variant G61A gene and hepatocellular carcinoma in pa-tients with chronic hepatitis B virus infection in China. Chin J Biologicals, 21, 1051- 3.

Qi P, Wang H, Chen YM, et al (2009). No association of EGF 5'UTR variant A61G and hepatocellular carcinoma in Chinese patients with chronic hepatitis B virus infection. Pathology, 41, 555-60.

Shahbazi M, Pravica V, Nasreen N, et al (2002). Association between functional polymorphism in EGF gene and malignant melanoma. Lancet, 359, 397-401.

Shi HZ, Ren P, Lu QJ, et al (2012). Association between EGF, TGF- $\beta 1$ and TNF- $\alpha$ gene polymorphisms and hepatocellular carcinoma. Asian Pac J Cancer Prev, 13, 6217-20.

Stoscheck CM, King LE Jr (1986). Role of epidermal growth factor in carcinogenesis. Cancer Res, 46, 1030-7.

Suenaga M, Yamada S, Fujii T, et al (2013). afunctional polymorphism in the epi-dermal growth factor gene predicts hepatocellular carcinoma risk in Japanese hepatitis cpatients. Onco Targets Ther, 6, 1805-12.

Tanabe KK, Lemoine A, Finkelstein DM, et al (2008). Epidermal growth factor gene functional polymorphism and the risk of hepatocellular carcinoma in patients with cirrhosis. JAMA, 299, 53-60.

Wang HX, Xie WM, Zhou GQ (2009). Epidermal growth factor gene polymorphism associated with susceptibility to hepatocellular carcinoma. Guangxi Medical Univer-sity. Master Dissertation.

Wu J, Zhang W, Xu A, et al (2013). Association of epidermal growth factor and epidermal growth factor receptor polymorphisms with the risk of hepatitis b vi-rus-related hepatocellular carcinoma in the population of north china. Genet Test Mol Biomarkers, 17, 595-600.

Yamaguchi K, Carr BI, Nalesnik MA (1995). Epidermal growth factor-induced hepa-tocellular carcinoma: gene expression profiles in precursor lesions, early stage and sol-itary tumours. J Surg Oncol, 58, 240-5.

Yang Z, Wu Q, Shi Y, et al (2012). Epidermal growth factor $61 \mathrm{~A}>\mathrm{G}$ polymorphism is associated with risk of hepatocellular carcinoma: a meta-analysis. Genet Test Mol Biomarkers, 16, 1086-91.

Yuan JM, Fan Y, Ognjanovic S, et al (2013). Genetic polymorphisms of epidermal growth factor in relation to risk of hepatocellular carcinoma: two case-control studies. BMC Gastroenterol, 18, 13, 32 .

Yue AM, Xie ZB, Guo SP, et al (2013). Implication of polymorphisms in DNA repair genes in prognosis of hepatocellular carcinoma. Asian Pac J Cancer Prev, 14, 355-8.

Zender L, Villanueva A, Tovar V, et al (2010). Cancer gene discovery in hepatocellu-lar carcinoma. J Hepatol, 52, 921-9.

Zintzaras E, Ioannidis JP (2005). Heterogeneity testing in meta-analysis of genome searches. Genetic epidemiology, 28, 123-37.
Zhong JH, You XM, Gong WF, et al (2012). Epidermal growth factor gene poly-morphism and risk of hepatocellular carcinoma: a meta-analysis. PLoS One, 7, 32159. 